\title{
Is the Mandatory Implementation of a Passport for COVID-19 Vaccine Reduce the Transmission or Not?
}

\section{Huang Wei Ling*}

Infectious Diseases, General Practice, Nutrition, Acupuncture and Pain

Management, Medical Acupuncture and Pain Management Clinic, Franca, São

Paulo, Brazil

*Corresponding Author: Huang Wei Ling, Infectious Diseases, General Practice, Nutrition, Acupuncture and Pain Management, Medical Acupuncture and Pain Management Clinic, Franca, São Paulo, Brazil.
Received: July 26, 2021

Published: August 12, 2021

(C) All rights are reserved by Huang Wei Ling.
The author will write on this topic on behalf of all people who are willing to preserve their health and are being forced to take the vaccine for COVID-19.

On this subject, several items have to be evaluated and not only referring to the pandemic that this measure will prohibit the spread of the virus or not [1].

One of the things that the author is sure of is that the measures taken are not to treat the cause because, according to the population we have these days, they are not the same as they were 6 or 7 years ago as she showed in the article entitled Is the Population in the World the Same as in the Past? [2].

In the article written by Speiser and Bachmann (2020), entitle COVID-19: Mechanisms of Vaccination and Immunity, they are saying that it is important to understand the capability to spread and biological mechanism of the virus. There are three priorities strategies that are: 1) continue the hygiene measurements and physical distancing, 2) viral monitoring (geographically and on in time) 3) rapidly increase the world's population immunity [3].

According to the article written by Huang (2021) entitled Are We Vaccinating Immunocompetent or Immunocompromised People for COVID 19? there was a change in the energy pattern of the patients that she was attending from 2015 to 2020 in which more than $90 \%$ of its patients in Brazil between evaluated was sud- denly without any energy in the chakras' energy centers (in 409 patients records from 1000 patient's measurements). According to traditional Chinese medicine, energy is linked to immunity and this means that the majority of the population she is attending is in a category of immunocompromised and not immune competent, as the majority of the doctors are thinking that they are today [1].

The cause of this lack of energy is related to electromagnetic waves of the $5 \mathrm{G}$ telecommunication telephony and with the modernization and speed with which Global communication has been carried out. Very few studies have been done in this area and the few studies that exist are always carried out by research cartels, saying that electromagnetic waves are not harmful to health $[1,4,5]$.

But in the article written by the author, she says that most people are immunodepressed due to the fact that they have low energy in the chakras' energy centers and according to traditional Chinese medicine energy means our immunity $[1,4,5]$.

In China, when a patient has a condition of energy deficiency in some organ, it is very common to use Chinese herbal therapy. But in the current case, most patients nowadays are low in general energy, this Chinese herbal medicine could also not be suitable for the type of patient that we have these days as it is considered a highly concentrated drug and could induce harm in the vital energy, according to Arndt Shultz Law [6]. 
Therefore, the author since 2015 has been studying homeopathy in Brazil and after this, she began to write another theory linking homeopathy with theories of traditional Chinese medicine that have been in existence for over 5,000 years. This theory written by the author (2020) is entitled Constitutional Homeopathy of theFive Elements Based on Traditional Chinese Medicine. According to Hippocrates, we should consider ancient medical traditions prior to the current medical practice nowadays $[7,8]$.

In this way the author is treating the lack of energy of the five massive organs or the chakras' energy centers using highly diluted medicines (homeopathies medications) and toning the energy production to maintain the health of the entire population, improving in this way, the immune system of our body that is deficient nowadays, showed by the author (2021) in the article Are We Vaccinating Immunocompetent or Immunocompromised People for COVID-19? $[1,7]$.

According to the article wrote by Huang (2021) entitled Are We Vaccinating Immunocompetent or Immunocompromised People for COVID 19?, she demonstrates that $97 \%$ of her patients in Brazil are deficient in Kidney energy which works like Zheng Qi, which is energy that protects the body against the invasion of external pathogenic factors. In the article wrote by Huang (2021) entitled Energy Alterations and Chakras' Energy Deficiencies and Propensity to SARS-CoV-2 Infection, the author is saying that SARS-CoV-2 is considered an invasion of external pathogenic factor according to traditional Chinese medicine, and in this article she is explaining that the majority of the population nowadays are weak in this energy that protects against this invasion of external pathogenic factor $[1,4]$.

In the article written by Huang (2021) entitled Are the Vaccines the Only Solution to Control COVID-19 Pandemic? the author is questioning if the vaccines will resolve our problems since the different perspectives in the view of the problem is only partial in Western medicine and it is not considering the energy deficiency that the entire population in the world is suffering, that all the problem are in the root level of this tree, that the majority of the doctors and scientists are not taking into account [9].

In the article written by Sonani., et al. (2021) entitled COVID-19 vaccination in immunocompromised patients, they are saying that patients in use of immunosuppressant medications needs special attention due to the fact that this kinds of patients was not enrolled in the vaccines' trial and was excluded. The efficacy of vaccines in this type of population still need to be evaluate because there is a decrease in the serological response after other vaccines [10].

In the article written by Anand and Stahel (2021) entitled $R e$ view the safety of Covid-19 mRNA vaccines: a review, they are saying that the rapid development of the COVID-19 vaccines' and due to the uncertainty of the potential adverse effects in long term raised some hesitation against the SARS-CoV-2 mRNA vaccine in the community globally. According to them, the success in the vaccination depends on three factors: 1) widespread access in vaccine under appropriate storage conditions; 2) sufficient number of vaccinators ;3) willingness of the population to be vaccinating [11].

In the article written by Huang (2021) entitled Are the Vaccines the Only Solution to Control COVID-19 Pandemic? the author mentioned the study made by Arvas (2014) that said that antibody levels in health individuals cannot be the same in the immune compromised category [9].

Using another type of therapy, using homeopathies medications to restore the lack of energy in the five massive organs (according to the theory Constitutional Homeopathy of the Five Elements Based on Traditional Chinese Medicine), will not have more risk of death or other complications, on the contrary, the population will be stronger and rejuvenate and will likely have fewer chronic diseases as the author has published in all her articles such as reduction in diabetes, in high blood pressure, in myocardial infarction chance, of having a stroke or even cancer and other problems, there will be more control of blood glucose and blood pressure [12-15].

There will also be less incidence of stroke and improvement of emotional illnesses such as anxiety, depression, panic syndrome, insomnia, which are diseases that most affect the world population today [15-17].

This method of the chakras' energy centers could be used in all patients who are included and who are not included in the vaccination schedule, which are pregnant women and patients, other patients who could not take the vaccine, patients who are using immunosuppressive drugs, and those patients who are having anaphylactic reaction to the components of vaccine [7]. 
What we learned from this pandemic is that it is necessary to integrate Western Medicine and Eastern medicine to understand more deeply how to prevent and treat this pathology, since the current population is different from what it was in the past ( 6 or 7 years ago) and there are other pandemics that are likely to be in an energy situation different from the current one. This phrase was written by Huang (2021) in the article entitled Is the Population in the World the Same as in the Past? [2].

In another article written by Zaporozhan and Ponomarenko (2010), entitled Mechanisms of Geomagnetic Field Influence on Gene Expression Using Influenza as a Model System: Basics of Physical Epidemiology 2010, they are saying that there is a role in the electromagnetic and geomagnetic field that can influence the flu epidemic process [18].

Noting the drug treatment recommendations for treating postvaccination symptoms and COVID-19 treatment protocols, if we pay attention to Arndt Schultz's law, created in 1888 by two German researchers, they say that highly concentrated drugs of any nature reduce the vital energy of the patient or it can even lead to death and highly diluted drugs improves vital energy [19].

Therefore, drugs that were previously considered unscientific by the medical community, are the drugs of choice for treatment of COVID-19 and for the treatment of all pathologies nowadays. Highly concentrated drugs should be left to be used only in cases where the use is indispensable to maintain the patient's life. This phrases were written by the Huang (2021) in the article Are the Medications that we are Prescribing to our Patients Harming them? and in the article also written by Huang (2021) entitled Why Medications Used Nowadays are Harmful to Our Health? [20].

In the article written by Doulberis., et al. (2021), entitled Does COVID-19 Vaccination Warrant the Classical Principle "ofelein i mi vlaptin"?, they are saying that the development of COVID-19 vaccines, due to the emergency, leads to incomplete evaluation despite the biological and intensive medical research. The accelerated vaccines development leads and downgraded the necessity of pre-clinical evaluation to evaluate harmful consequences and medium and long term consequences. They are also saying that the high SARSCoV-2 potential mutations and aggregated genetical alterations, provoke an uncertainty concerning the vaccines efficacy against dominant strains and clinical immunity [21].
According to Doulberis., et al. (2021), vaccination in the context of the anti-COVID-19 vaccine is a global experience and for this reason, global anti-vaccination actions is increasing day-by-day. In this article, they are affirming that individual autonomy is fundamental and it does not justify obligatory vaccination. The individual needs to live according to what they consider healthy and there is a need to keep preserved this plans. And our society prohibits slaves but they are saying that our society is killing the individuals and slaving them to induce a do something that is incompatible to their idea to have a health life [21].

Also, in this article they are saying that the possibility of vaccine's induce auto-immunity including Guillian Barre syndrome, demyelinating neuropathies, narcolepsy, postural orthostatic tachycardia syndrome, multiple sclerosis, in some groups susceptible a it. For this reason, one of the side effects of mass vaccination program could be an autoimmune disease especially in persons that is genetically prone to autoimmune disease [21].

According to them, the universal immune passport in the lack of accurate studies could lead to enormous cost on the entire community of the planet, increasing the auto-immune disorders and the time of suffering after the development of this disease [21].

The development a vaccine for one disease may take years (about 10 to 15 years) to be commercially available. The estimate risk failure is about $94 \%$ or high. The development of vaccine for Middle East Respiratory Syndrome (MERS) and SARS related research do not reach a successful vaccine after decade of study and efforts [21].

In the talk of a volcanologist and pharmaceutical executive, Stanley Plotkin, stated that "he is waiting for further data concerning T-cells responses and all long the duration of antibodies will occur" [21].

In the article wriiten by Huang (2021) entitled) Are We Vaccinating Immunocompetent or Immunocompromised People for COVID-19?, the author is saying that the majority of our population nowadays is classified as immune deficient due to the lack of energy in the chakras' energy centers. The chakra that vis responsible for the production of Blood cells is the fifth and the author demonstrated that the majority, quite $97 \%$ of the patients do not have energy in this chakra, due to the influences of the electromagnetic 
waves in our life. This lack of energy could cause a lack of production of blood cells including B and T cells for the production of antibodies after vaccination $[1,9]$.

In the article written by Norbert at al. (2018) in a recent nature state art of review, they concerns about the efficacy about this vaccine because they are stressing the ability of the vaccine to activate germinal B cells response and T cells follicular helper, and they do not know why they cannot understand the response of this vaccine to activate this cells. They also comment that there are no regulatory guidance by EMA or FDA to rule about human mRNA vaccine [22].

According to the director of the National Institute of Allergy and infectious disease, Professor Antony Fauci, there is no evidence yet that the vaccines can stop the transmission and replace the social distancing and masking measurements [21].

In the article written by Doulberis., et al. (2021), they said that new technology can somethimes cause unexpected side effects and problems [21].

In the study made by Mayo Clinic by Stiepan (August 2021) entitled COVID-19 infection among vaccinated people: What you need to know, they are affirming that even vaccinating people can spread COVID-19. In the interview with Dr. John O'Horo, infectious disease specialist in Mayo Clinic, he is emphasizing the importance to the people to know that people who is vaccinated by COVID-19 can transmit and potential to have infections by SARS-CoV-2. In this study, they are saying that even fully vaccinating people still have the potential to transmit COVID-19 to others. They recommend that people still continue using mask even after vaccination and these people can have high levels of virus in the nose and in their throat (even asymptomatic). They are also saying that many types of vaccines can prevent illness but not necessary prevent the transmission of illness to others [23].

In an article written by Huang (2021) entiled Is the Medication used in Intubation of Patients with Covid-19 Affecting the Outcome of the Patient's Treatment?, the author questioned whether virusrelated deaths are actually caused by the virus or by the use of medications used for the treatment of these patients and which are in the protocols of many health institutions [24].
From the studies that the author has carried out for several years being one of the specialties in infectious and parasitic diseases, she has also treated most infectious diseases without the use of antibiotics and she has published several articles on treatment of community and hospital infectious, the majority of them treating without the use of any antibiotics. The author realizes that perhaps there is the relationship of the adhesiveness of COVID-19 in patients who have internal Heat formation, generated when there is internal energy deficiency, according to traditional Chinese medicine reasoning $[25,26]$.

This association, the author has demonstrated in several articles published in medical journals reporting increased bacterial activity by bladder cells, for example, when there is internal Heat formation by patients generated by energy deficiency [25-27].

Also not all cases of nosocomial infection in which the author was able to treat all the cases in which the patients had infection with multidrug-resistant bacteria. The only thing that was done in the treatment, besides internal energy rebalancing, guiding the diet by Chinese medicine is the apex ear bloodletting in which through this procedure it removes the internal Heat in the patient and balancing Yin, Yang, Qi and Blood energies using auricular acupuncture with mustard seeds. It removes the Heat and rebalances the energy and the bacteria could not adhere anymore and the author was able to promptly eradicate the infection without using antibiotics [25-27].

In the treatment of all patients with lack of energy, there will be less formation of internal Heat and thus the virus will have no way of fixing cells and so people are not prone to infection by COVID-19 [28].

As the author always emphasizes in all her presentations, Western medicine treats at the leaf level and Eastern medicine treats the root level [12, 29].

The integration of the two medicines is currently of fundamental importance for an in-depth understanding of the causes and factors that explain the transmission of the disease and that are not only caused by the virus, and also the exposition to external pathogenic factors (Cold, Wind, Heat, Humidity, Dryness) and the energy that the patient have before acquiring COVID-19. There is 
a relationship between the Zheng-Qi (the energy that protects our body against the invasion of this pathogenic factors and the Xie-Qi, that is the pathogenic factor), When the Zheng-Qi is high and the $X i e-Q i$ is low, the patient will not be sick after exposition to the virus itself but when the Zheng-Qi is low and the Xie-Qi is bigger or in the same highty, the patient will be sick with the exposition to the virus (and this is the situation that we are facing now, as the Zhen Qi of the quite entire population is low, as demonstrated by the author In the article Are We Vaccinating Immunocompetent or Immunocompromised People for COVID-19?) [1,4].

Unlike the authors and professionals who study the vaccine, we are currently vaccinating immunocompromised people and therefore the immunogenicity, the production of antibodies in this population will be fatally harmed if the vaccines are applied to current populations that still suffering interference from electromagnetic factors because they will maintain the low level of energy leading to the low production of antibodies [1,9].

Medicines considered scientific, such as the use of anti-inflammatory, antibiotics and all classes of highly concentrated medicines can be dangerous for the use in the patients nowadays (Arndt Shultz Law) as the author showed in the article written by her (2021) entitled Is the Population in the World the Same as in the Past? [2,19].

The use of any medications in high concentrations nowadays can harm the vital energy and can worsen the evolution and treatment of COVID-19 and should be avoided in this current population, especially during the acute treatment of COVID-19, as demonstrated in the article written by the author entitled Is the Medication used in Intubation of Patients with Covid-19 Affecting the Outcome of the Patient's Treatment? [2, 24].

The lack of a complete vision of the whole can make the authorities make decisions that are not adequate for the current reality and stop using less safe measures than those that could be used, for example, with the use of highly diluted drugs called SARS-CoV-2 nosodes and with fewer side effects than those currently used because the vaccines were made without an adequate study and with possible cause of very serious effects in the uncertain future and are considered highly concentrated medications, so it can cause the same effects of lowering the vital energy of the patient [21, 30, 31].

With the measures currently taken against the COVID-19 pandemic, deeper measures reaching the cause must be implemented and treatments to replenish the energy deficiency with highly diluted medicines, according to the theory Constitutional Homeopathy of the Five Elements based on Traditional Chinese Medicine must be applied widely as they are safe medicines, with no side effects, without high cost and that, in addition to treating the lack of immunity, they will reduce the formation of all chronic diseases, unlike when you use the vaccine, it increases the chance of the person having serious side effects such as the induction of auto-immunity diseases or even death as the worst side effect $(53,4 \%$ per million vaccination), as demonstrated in the article written by Lv (2021) entitled Mortality Rate and Characteristics of Deaths Following COVID-19 Vaccination $[4,7,21,32]$.

\section{Bibliography}

1. Huang WL. "Are We Vaccinating Immunocompetent or Immunocompromised People for COVID 19?" Journal of Vaccines Research and Vaccination 7 (2021): 018.

2. Huang Wei Ling. "Is the Population in the World the Same as in the Past?". Acta Scientific Clinical Case Reports 2.6 (2021).

3. Speiser D and Bachmann M. "COVID-19: Mechanisms of Vaccination and Immunity". Vaccines (Basel) 8.3 (2020): 404.

4. Huang Wei Ling. "Energy Alterations and Chakras' Energy Deficiencies and Propensity to SARS-CoV-2 Infection". Acta Scientific Microbiology 4.4 (2021): 167-196.

5. Hardell L and Carlberg M. "Health risks from radiofrequency radiation, including $5 \mathrm{G}$, should be assessed by experts with no conflicts of interest". Oncology Letter 20.4 (2020).

6. Lo DC. "Chinese masterful formulas". 1st editio. São Paulo: Roca (2007): 608.

7. Huang WL. "Constitutional Homeopathy of the Five Elements based on Traditional Chinese Medicine". Acta Scientific Medical Sciences 4.7 (2020): 57-69.

8. Craik E. "The "Hippocratic" Corpus: Content and Context". Routledge (2014): 344.

9. Huang Wei Ling. "Are the Vaccines the Only Solution to Control COVID-19 Pandemic?". Vaccines 6.1 (2021): 000152.

10. Sonani B., et al. COVID-19 vaccination in immunocompromised patients". Clinical Rheumatology (2021): 1-2. 
11. Anand P and Stahel VP. "The safety of Covid-19 mRNA vaccines: a review". Patient Safety Surgery 15 (2021): 20 (2021).

12. Huang WL. "Why Are Diabetic Patients Still Having Hyperglycemia despite Diet Regulation, Antiglycemic Medication and Insulin?" International Journal of Diabetes and Metabolic Disorder 4.2 (2019): 1-14.

13. Huang WL. "The Importance of Correcting Energy Imbalances in the Prevention and Treatment of Myocardial Infarction". Acta Scientific Medical Sciences 4.6 (2020): 20-27.

14. Huang WL. "The Importance of Correcting Energy Imbalances in the Prevention and Treatment of Câncer". Advances in Cancer Research and Clinical Imaging 3.1 (2020): 1-10.

15. Huang WL. "Why Do Patients Still have Anxiety Symptoms Despite the Use of Psychotropic Medications". General Medicine: Open Access 2.2 (2019): 13-23.

16. Huang WL. "Energy Disturbances in Panic Syndrome and How Can we Treat it in Adolescence?" Journal of Neurology and EXperimental Neural Science 3.1 (2020): 139-49.

17. Huang WL. "Auricular Acupuncture and Chinese Dietary Counselling in the Treatment of Insomnia". Archives of Neurology and Neuro Disorders 3.1 (2020): 1-11.

18. Zaporozhan V and Ponomarenko A. "Mechanisms of Geomagnetic Field Influence on Gene Expression Using Influenza as a Model System: Basics of Physical Epidemiology". International Journal of Environmental Research and Public Health 7.3 (2010): 938-965.

19. LEESER 0. "Support of homeopathy by the Arndt-Schulz law". National Center for Biotechnology Information. 1953/ Jul 31 (1953).

20. Huang WL. "Are the Medications that we are Prescribing to our Patients Harming them?" ARC Journal of Orthopedics 6.1 (2021): 11-13.

21. Doulberis M., et al. "Does COVID-19 Vaccination Warrant the Classical Principle "ofelein i mi vlaptin?" Medicina (Kaunas) 57.3 (2021): 253.

22. Pardi, N., et al. mRNA vaccines - a new era in vaccinology. Nat Rev Drug Discov 17 (2018): 261-279 https://doi. org/10.1038/nrd.2017.243.
23. COVID-19 infection among vaccinated people: What you need to know. Mayo Clinic. August 5, 2021. Retrieved 08/10/2021. Available: https://newsnetwork.mayoclinic.org/discussion/ covid-19-infection-among-vaccinated-people-what-you-needto-know/

24. Huang Wei Ling. Is the Medication used in Intubation of Patients with Covid-19 Affecting the Outcome of the Patient's Treatment?. Archives of Anesthesiology. 2021; 4(1): 01-03

25. Huang WL. "Is it Possible to Treat Community-Acquired and Nosocomial Infections with the Same Method Without the Use of Antibiotic?" Journal of Applied Microbiology 2.2 (2019): $1-13$.

26. Huang WL. "Energies Alterations and Chakras Energies Deficiencies as the Main Cause of Recurrent Urinary Tract Infection Resistant to Antibiotics Treatments". Clinical Research in Urology 3.2 (2020): 1-8.

27. Huang WL. "Can Hospital Osteomyelitis Be Treated Without the Use of Antibiotics?" Acta Scientific Microbiology 1.9 (2018): 4-12

28. Huang WL. "How Can we Treat Recurrent Herpes Virus Infection Without the Use of Antiviral Drugs?". Acta Scientific Medical Sciences 3.8 (2019): 152-159.

29. Huang WL. "Why do Patients Still Catch Hospital Infections Despite the Practice of Infection Prevention and Control Programs?" Acta Scientific Microbiology 1.4 (2018): 34-43.

30. Kalliantas D et al. Homeopathy combat against coronavirus disease (Covid-19). Z Gesundh Wiss. 2020 Jun 5 : 1-4. doi: $10.1007 / \mathrm{s} 10389-020-01305-\mathrm{z}$

31. Mukherjee S et al. Preparation of Coronavirus nosodes sourced from a clinical sample of SARS-Cov-2 positive patient, inactivated strain, and spike glycoprotein. Pharmaceutical Research. 2021-03-14. DOI: https://doi.org/10.51910/ijhdr.v19i4.1054

32. Gang Lv et al. Mortality Rate and Characteristics of Deaths Following COVID-19 Vaccination. Front Med (Lausanne). 2021 May 14;8:670370. doi: 10.3389/fmed.2021.670370. eCollection 2021

\section{Volume 4 Issue 9 September 2021} (C) All rights are reserved by Huang Wei Ling. 